\title{
Novel Formula for Calculation of the Optimal Insertion Depth for Cuffed Endotracheal Tubes in Pediatric Major Surgery
}

\author{
Mao Kinoshita, Kazuma Hayase, Mizuki Bando, Naofumi Kawai, Masaru Shimizu, \\ Masayuki Shibasaki*
}

Department of Anesthesiology, Kyoto Prefectural University of Medicine, Kamigyo, Kyoto, Japan

Email: *mshiba@koto.kpu-m.ac.jp

How to cite this paper: Kinoshita, M., Hayase, K., Bando, M., Kawai, N., Shimizu, M. and Shibasaki, M. (2019) Novel Formula for Calculation of the Optimal Insertion Depth for Cuffed Endotracheal Tubes in Pediatric Major Surgery. Open Journal of Anesthesiology, 9, 42-50. https://doi.org/10.4236/ojanes.2019.93005

Received: February 14, 2019

Accepted: March 16, 2019

Published: March 19, 2019

Copyright $\odot 2019$ by author(s) and Scientific Research Publishing Inc. This work is licensed under the Creative Commons Attribution International License (CC BY 4.0).

http://creativecommons.org/licenses/by/4.0/

\section{(c) (i) Open Access}

\begin{abstract}
Background: Accurate determination of the optimal insertion depth of a pediatric endotracheal tube (ETT) is quite important. The aim of this study was to create an easily available formula that can be used to determine the optimal insertion depth for a cuffed ETT even without depth marking with clear definitions of the upper and lower limits for the tip of ETT in the trachea in clinical practice. Methods: Eighty children under 12 years of age were enrolled. The depth marking of the cuffed ETT was placed at the vocal cords and both lungs were then auscultated using a stethoscope. The upper limit was radiographically defined as the position of the tip of the cuffed ETT being between the clavicles. The lower limit was defined as a distance of $5 \mathrm{~mm}$ above the carina. The relationship between the insertion depth and patient characteristics was analyzed to create a formula for optimal ETT insertion depth. Results: Sixty-nine ETTs were optimally placed in the trachea. There were good correlations between the optimal insertion depth of ETTs and patients characteristics (height $(\mathrm{R}=0.92)$; BSA $(\mathrm{R}=0.92)$; weight $(\mathrm{R}=0.91)$; age $(\mathrm{R}=0.88)$ ). Using these patient characteristics, we created the following three formulas for calculation of the optimal insertion depth for pediatric cuffed ETTs: insertion depth $(\mathrm{cm})=$ height $(\mathrm{cm}) / 11+5.5$, weight $(\mathrm{kg}) / 3+9.5$ or $11+3 / 4 \times$ age (years). The rates of appropriate tube placement of both pediatric cuffed ETTs were $87.5 \%$ (Hi-Contour) and 85.0\% (Microcuff). Conclusions: Our formula and graphs may be easy to determine the optimal insertion depth of cuffed ETT even without depth marking in clinical practice.
\end{abstract}

\section{Keywords}

Insertion Depth, Infants, Small Children, Cuffed Endotracheal Tubes 


\section{Introduction}

Pediatric endotracheal tube (ETT) management is of great concern in anesthesia and intensive care medicine. Prediction of the optimal size of the tube and its insertion depth are considerable challenges, especially in infants and small children [1] [2] [3]. Uncuffed ETTs had been used for several decades [4] and the many reports on the advantages of cuffed ETTs and improvements in the cuffs available had led to the use of cuffed ETTs even in infants and small children [5] [6] [7]. Instead of widespread of cuffed ETTs, shortcomings of cuffed ETTs were reported including cuff positions and depth markings [8]. Cuff positions and location of depth markings vary from brand to brand of ETT, even among those having the same internal diameter. Some pediatric cuffed ETTs have no depth marking, and others have multiple depth markings. The length of the trachea in pediatric patients is shorter than that in adult patients. Anesthesiologists need to take into account the distance between the carina and the vocal cords to avoid endobronchial intubation and the cuff coming into contact with the vocal cords. The cuff also avoiding subglottic area, should be placed below the lower border of the cricoid. Several methods for prediction of the optimal insertion depth have been reported [9] [10] [11] [12] [13]; however, none of these reports has clearly defined the upper and lower limits of the tube tip in the trachea. Therefore, we defined that the definition of upper and lower limits of the tube tip in the trachea is based on the tip of the endotracheal tube which is 5 $\mathrm{mm}$ head side from the tracheal bifurcation and between bilateral clavicle mesial lines [14]. Furthermore, only one brand of ETT was used in some of these reports. A recent report has tested several tube brands and insertion formulas including position of the cuff within the trachea [15]. The aim of this study is to create a formula for optimal depth of insertion of a pediatric cuffed ETT that includes both the upper and lower limits for the tube tip in the trachea.

\section{Patients and Methods}

\subsection{Study Design and Participants}

The study was approved by the University's Institutional Review Board of Kyoto Prefectural University of Medicine (ERB-C-344) and written informed consent was obtained from all the patients' parents or guardians. The trial was registered prior to patient enrollment at the UMIN Clinical Trials Registry (http://www.umin.ac.jp/english/) (UMIN000016324, Principal investigator: Teiji Sawa, Date of registration: February, $\left.1^{\text {st }}, 2015\right)$. From September in 2015 to March in 2018, eighty children under the age of 12 years who required general anesthesia with endotracheal intubation were enrolled. The inclusion criteria were pediatric patients required general anesthesia with intubation and chest $\mathrm{X}$-ray for clinical purpose. The exclusion criteria were a disorder affecting the airway (e.g., tracheostenosis or tracheomalacia) or a history of difficult airway management. There were no patient groups affecting height or weight such as obesity, achondroplasia. 


\subsection{Study Protocol}

Cuffed ETTs were inserted after induction of general anesthesia with intravenous midazolam or thiopental and rocuronium bromide. The Hi-Contour (Mallinckrodt Pharmaceuticals, St Louis, MO, USA) and Microcuff (Halyard Health Care, Alpharetta, GA, USA) ETTs were randomly selected for use in this study. Initial size of cuffed ETT was selected by anesthesiologist. After that, the final size of cuffed ETT was determined by the air leak method. The air leak was checked with the cuff deflated when the lungs were inflated. If air leak was present at an airway pressure of $20 \mathrm{~cm} \mathrm{H}_{2} \mathrm{O}$, the cuffed ETT was changed to a larger size. If air leak was absent at an airway pressure of $30 \mathrm{~cm} \mathrm{H}_{2} \mathrm{O}$, the cuffed ETT was changed to a smaller size. The depth marking on the cuffed ETT was placed at the vocal cords and auscultation of both lungs was then performed using a stethoscope. Tracheal intubation was performed by an experienced anesthesiologist. Anesthesiologists confirmed depth marking of endotracheal tube was placed at the vocal cords using direct laryngoscopy. Depth marking was directly confirmed by an anesthesiologist. When using a Hi-Contour tube with an internal diameter $>3.5 \mathrm{~mm}$, the center between the two depth markings was placed at the vocal cords. Next, the distance between the carina and the tip of the cuffed ETT was measured using a flexible optical fiberscope. We defined both the upper and lower limits of the tip of the cuffed ETT to avoid damage to the vocal cords and accidental endobronchial intubation. The upper limit was radiographically defined as the position of the tip of the cuffed ETT when it was between the clavicles. The lower limit was defined as a distance of $5 \mathrm{~mm}$ between the carina and the tip of the cuffed ETT. The depth of insertion at a lip corner and the height, age, and weight of the patient were recorded. When the ETT was appropriately inserted; these data were then used to devise a formula for optimal depth of insertion of a pediatric cuffed ETT.

\subsection{Statistical Analysis}

The data are presented as the number and mean as appropriate. Quantitative data were analyzed using the paired-samples Student's $t$-test. Categorical variables were analyzed using the chi-squared test. A correlation analysis was performed to determine the relationships between insertion depth and patient height, body surface area (BSA), and age on Spearman's correlation analysis. An expression was created by simple linear regression analysis. A P-value $<0.05$ was considered statistically significant.

\section{Results}

Eighty children (31 female, 49 male) with a median age of $1.2(0.1-11.2)$ years were included in the study. There were no significant differences in demographics or clinical characteristics between the patients who were intubated with the Hi-Contour and those who were intubated using the Microcuff, (Table 1). An overview of the study protocol is shown in Figure 1. The distance between the carina and the tip of the cuffed ETT was not measured in two cases. The rates of 
Table 1. Patient characteristics by Microcuff vs. Hi-Contour group ( $\mathrm{n}=80$ patients).

\begin{tabular}{ccccc}
\hline & Range & Microcuff $(\mathrm{n}=40)$ & Hi-Countour $(\mathrm{n}=40)$ & P Value \\
\hline Age (years) & $0.1-11.2$ & $0.95(0.45,2.2)$ & $1.16(0.53,4.5)$ & 0.149 \\
Height $(\mathrm{cm})$ & $47.5-140.0$ & $71.0(61.0,83.5)$ & $74.1(65.9,100.2)$ & 0.196 \\
Weight $(\mathrm{kg})$ & $2.7-28.4$ & $7.7(5.5,11.7)$ & $9.7(6.1,16.6)$ & 0.10 \\
\hline
\end{tabular}

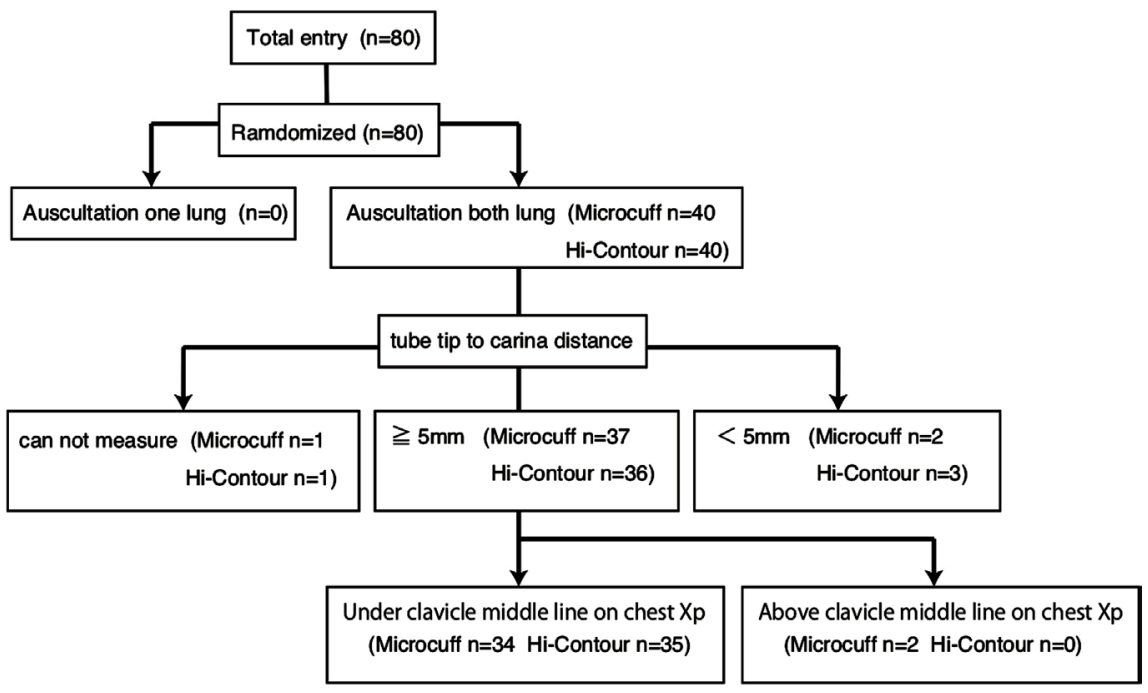

Figure 1. Study flow chart.

appropriate tube placement using depth markings were $87.5 \%$ for the Hi-Contour device and $85.0 \%$ for the Microcuff. The percent prevalence of the tip of the cuffed ETT is shown in Figure 2. The distance between the carina and the tip of the cuffed ETT ranged from $2.0 \mathrm{~mm}$ to $21.0 \mathrm{~mm}$ (Figure 3). The insertion depth correlated well with patient height $(\mathrm{R}=0.92, \mathrm{p}<0.001)$, BSA $(\mathrm{R}=$ $0.92, \mathrm{p}<0.001)$, weight $(\mathrm{R}=0.91, \mathrm{p}<0.001)$, and age $(\mathrm{R}=0.88, \mathrm{p}<0.001$; Figures $4(\mathrm{a})-(\mathrm{d})$, Table 2$)$.

The height-based formula for optimal insertion depth was as follows: insertion depth $(\mathrm{cm})=5.3954+0.092096 \times$ height $(\mathrm{cm})\left(^{*}\right)$. The weight-based formula for optimal insertion depth was as follows: insertion depth $(\mathrm{cm})=9.3343+$ $0.32245 \times$ weight $(\mathrm{kg})$. The age-based formula for optimal insertion depth was as follows: insertion depth $(\mathrm{cm})=11.035+0.73976 \times$ age (years).

Based on the above relationships, we propose the following formulas for calculation of the optimal depth of insertion of a cuffed ETT in infants and small children: insertion depth $(\mathrm{cm})=$ height $(\mathrm{cm}) / 11+5.5$; insertion depth $(\mathrm{cm})=$ weight $(\mathrm{kg}) / 3+9.5$; or insertion depth $(\mathrm{cm})=11+3 / 4 \times$ age (years).

\section{Discussion}

We have demonstrated the appropriate insertion depth of two types of pediatric cuffed ETTs using definitions of both the upper and lower limits of the tips. It is important to define both the upper and lower limits when using a cuffed ETT in a pediatric patient. A previous report showed that auscultation of both lungs 


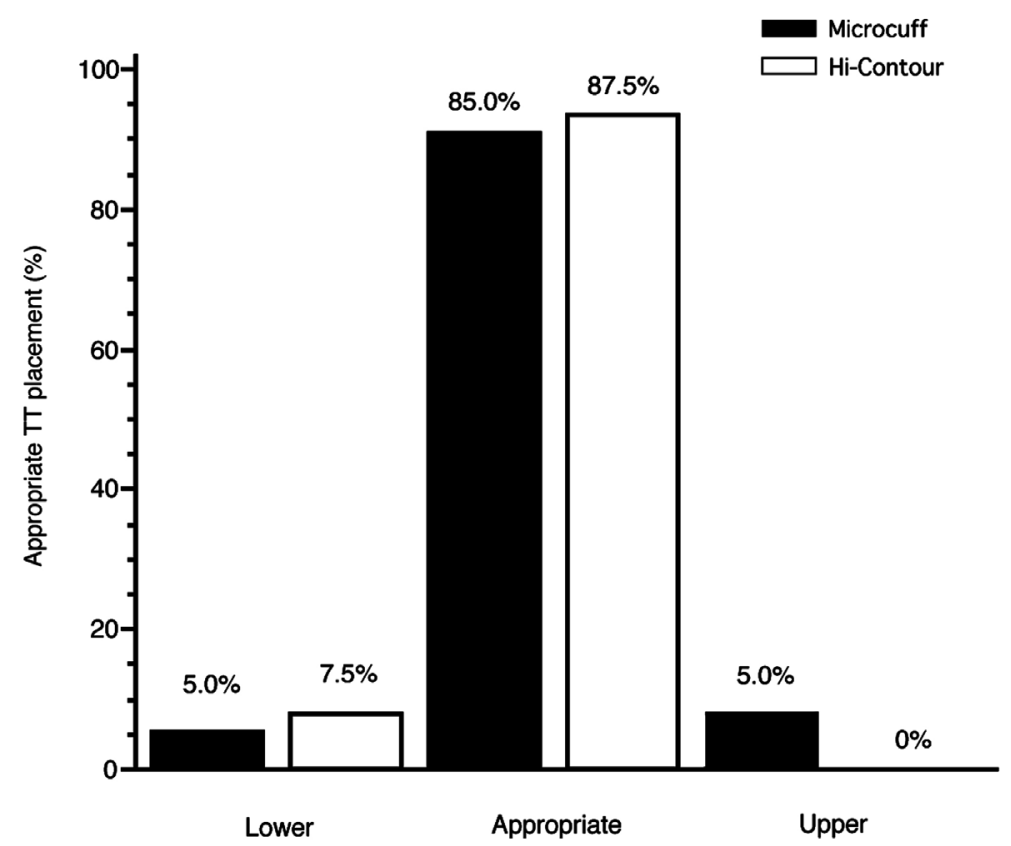

Figure 2. Prevalence of lower, appropriate or upper placement for each type of cuffed endotracheal tube.

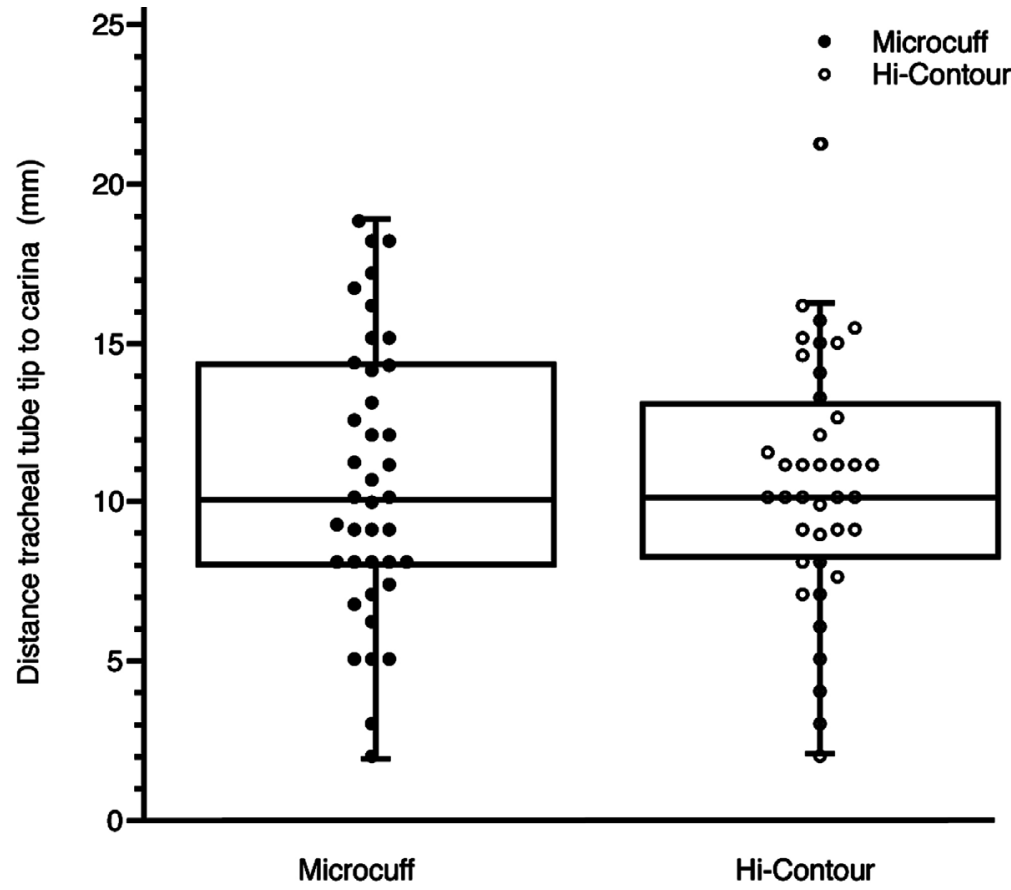

Figure 3. Distribution of the distance between the carina and the tip of the endotracheal tube using depth markings. The data are shown as the mean, 25th and 75th percentile, and range.

could not rule out endobronchial intubation in children [16]. The authors of that report discussed bilateral lung sounds owing to the Murphy eyes. Some ETTs, e.g., the Hi-Contour tubes, have a Murphy eye, and when used, the tip of the ETT might be placed very close to the carina even though both lung sounds 


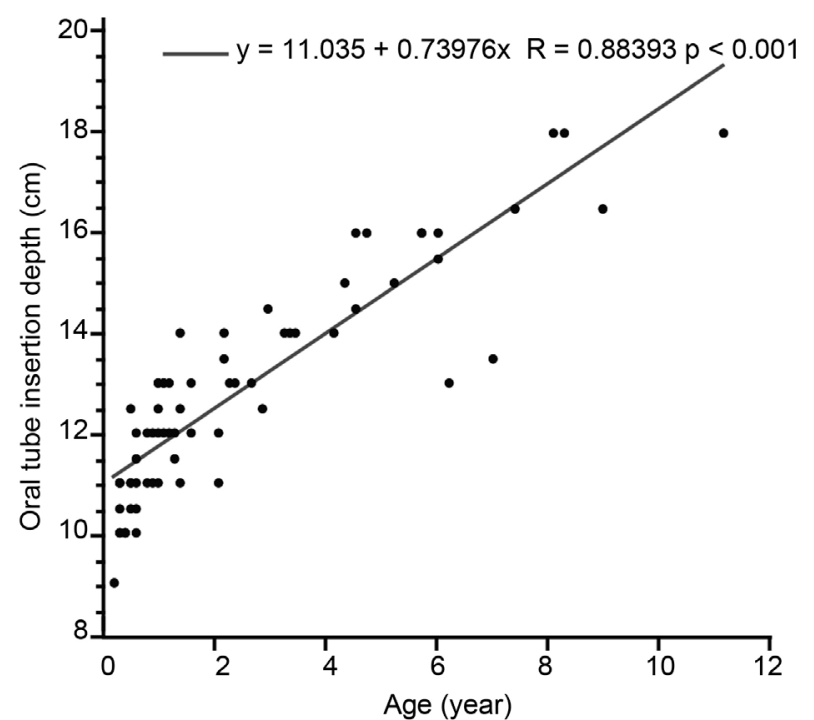

(a)

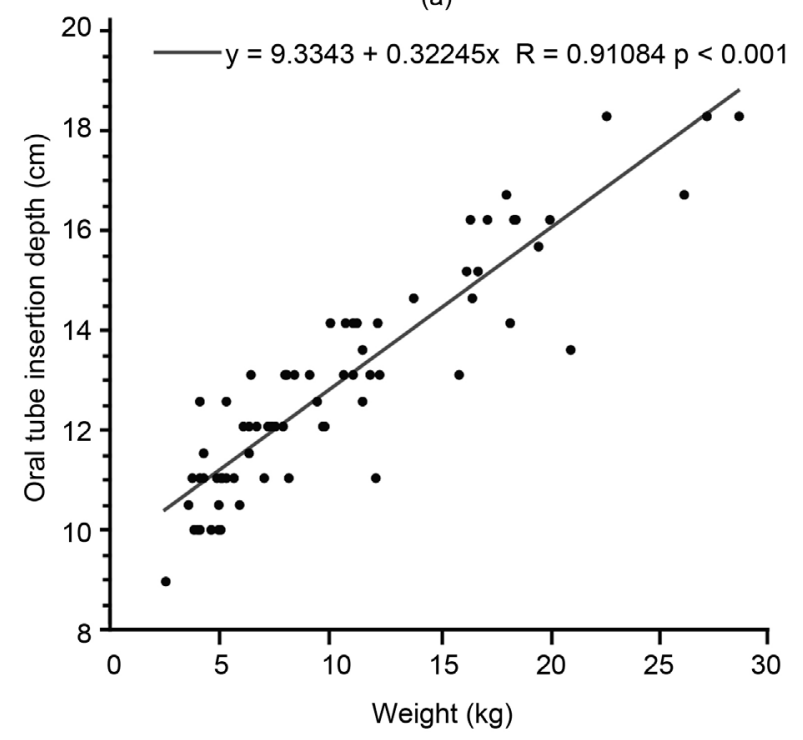

(c)

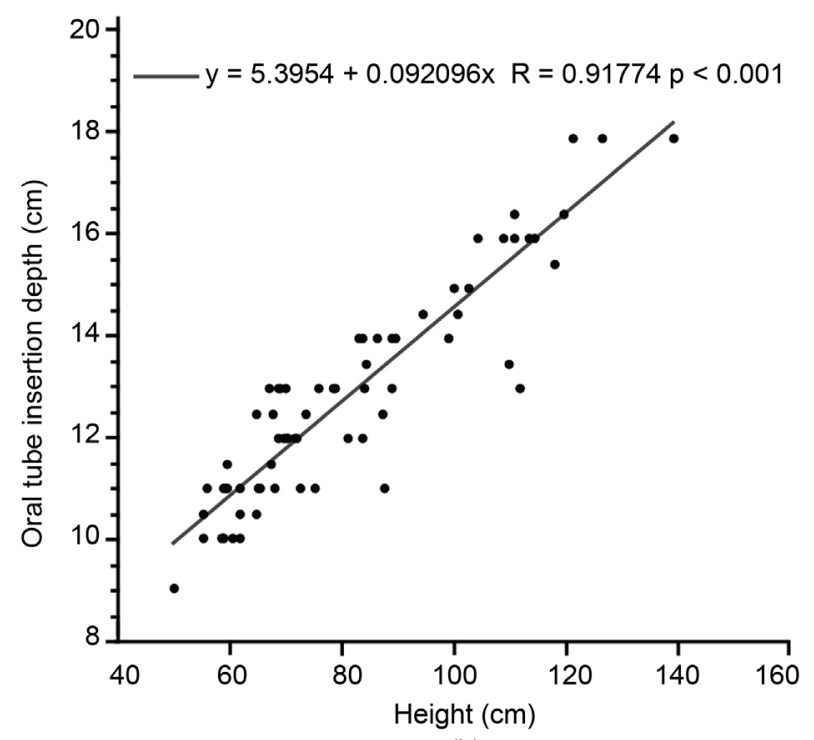

(b)

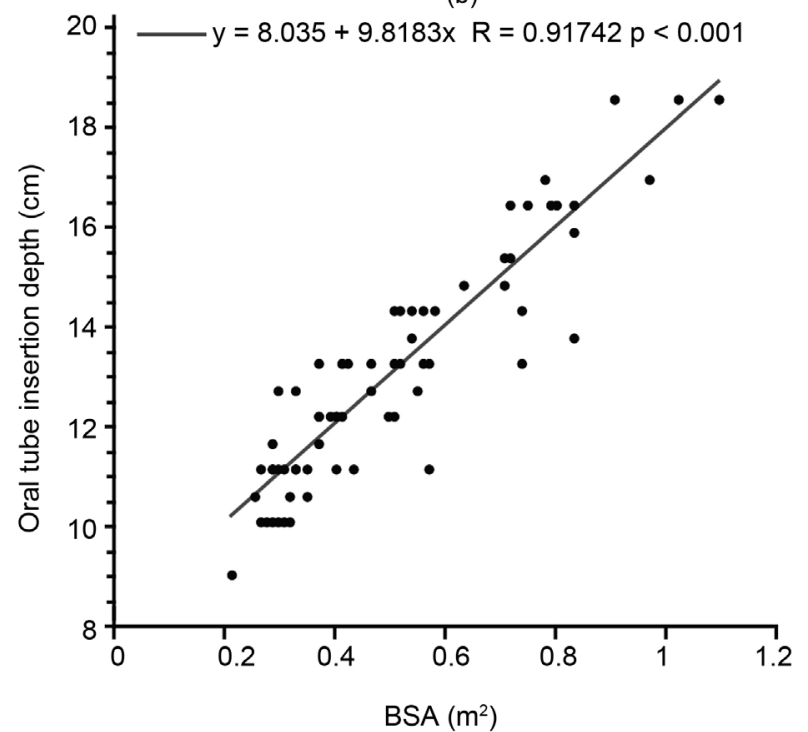

(d)

Figure 4. Linear regression plots comparing the insertion depth with the age, height, weight, and body surface area of the patient.

Table 2. Regression coefficient and 95\% confidence interval.

\begin{tabular}{ccccc}
\hline & & Regression coefficient & P5\% CI & $<$ Value \\
\hline \multirow{2}{*}{ Age (years) } & Slope & 0.741 & $0.645-0.837$ & $<0.0001$ \\
& Intercept & 11.040 & $10.704-11.376$ & $<0.0001$ \\
Height $(\mathrm{cm})$ & Slope & 0.092 & $0.082-0.102$ & $<0.0001$ \\
& Intercept & 5.395 & $4.584-6.206$ & $<0.0001$ \\
Weight $(\mathrm{kg})$ & Slope & 0.322 & $0.287-0.358$ & $<0.0001$ \\
& Intercept & 9.337 & $8.895-9.779$ & $<0.0001$ \\
\hline
\end{tabular}

are detected using a stethoscope. Furthermore, the cuffs should not be placed too close to the vocal cords to avoid damage. The proximal side of the cuff was placed 
caudal to the vocal cords in this study. Placement of the tip of the cuffed ETT more caudal than the clavicles was considered appropriate. The upper limit of the ETT tip was confirmed radiographically. Both types of ETT used in this study have depth markings, but some ETTs do not. Insertion depth of cuffed ETTs without depth marking cannot be determined by depth marking method. Insertion depth should be determined by other methods such as using height-based or weight-based formulas in case of cuffed ETTs without depth marking. However, these formulas were not created with the clear definitions of both the upper and lower limits of the tip of ETTs. Both the upper and lower limits of the cuffed ETT tip are needed when using a general formula even when the tube has no depth markings. The ratio of appropriate insertion depth for both the types of cuffed ETT used in this study was at least $80 \%$. Both tubes are well designed in terms of their depth markings. However, more than $10 \%$ of the tubes were placed too shallow or too deep. Hi-Contour tubes with an internal diameter > $3.5 \mathrm{~mm}$ have two depth markings whereas the Microcuff tubes have only one depth marking. In the present study, the center of the depth markings on the Hi-Contour tubes was placed at the vocal cords. The tips of these tubes might be too shallowly placed when using the distal depth marking and too deeply placed when using the proximal depth marking. It is very confusing which depth markings should be placed at the vocal cords in case of cuffed ETTs with multiple depth markings.

The insertion depth correlated well with height, BSA, weight, and age, indicating that the optimal insertion depth of ETTs could be determined using only one of these parameters. A previous report showed that height was the best predictor of optimal insertion depth for an ETT. Our results are consistent with that report. However, weight and age were also good predictors of optimal insertion depth of ETTs in our study. It is likely that these patient characteristics were good predictors of the optimal insertion depth because of the clearly defined upper and lower limits of the tip of the cuffed ETT and because of the creation of a general formula to predict the optimal insertion depth using data only for cuffed ETTs that were placed appropriately. Optimal insertion depth can be easily predicted after installation of all of our formula on electrical anesthesia record. Moreover, graphs in this study can make us determine the optimal insertion depth of cuffed ETTs even when electrical anesthesia records are not available.

The main limitation of this study is that the cuffed ETTs supplied by the various manufacturers have different characteristics, e.g., different cuff positions. [8] Further studies are required to validate our formula using brands of cuffed ETTs other than those used in the present study. It has been reported that the relative position of the ETT changes when the head is extended or flexed and during pneumoperitoneum in laparoscopic surgery [17] [18]. How head position and pneumoperitoneum might affect the measurements obtained using various methods for determination of insertion length is still unclear. 


\section{Conclusion}

In conclusion, it is clinically important to determine the optimal insertion depth of cuffed ETTs in children. We have created formulas for calculation of the optimal insertion depth of cuffed ETTs based on definitions of the upper and lower limits. Optimal insertion depth can be easily calculated by all of our formulas on electrical anesthesia record. Moreover, graphs in this study can make us determine the optimal insertion depth of cuffed ETTs even when electrical anesthesia records are not available.

\section{Disclosures}

This research did not receive any specific grant from funding agencies in the public.

\section{Conflicts of Interest}

No authors have any conflicts of interest or disclosures with regard to this study.

\section{References}

[1] Cole, F. (1957) Pediatric Formulas for the Anesthesiologist. The American Journal of Diseases of Children, 94, 672-673. https://doi.org/10.1001/archpedi.1957.04030070084009

[2] Khine, H.H., Corddry, D.H., Kettrick, R.G., Martin, T.M., McCloskey, J.J., Rose, J.B., et al. (1997) Comparison of Cuffed and Uncuffed Endotracheal Tubes in Young Children during General Anesthesia. Anesthesiology, 86, 627-631. https://doi.org/10.1097/00000542-199703000-00015

[3] Mariano, E.R., Ramamoorthy, C., Chu, L.F., Chen, M. and Hammer, G.B. (2005) A Comparison of Three Methods for Estimating Appropriate Tracheal Tube Depth in Children. Pediatric Anesthesia, 15, 846-851. https://doi.org/10.1111/j.1460-9592.2005.01577.x

[4] Orliaguet, G.A., Renaud, E., Lejay, M., Meyer, P.G., Schmautz, E., Telion, C., et al. (2001) Postal Survey of Cuffed or Uncuffed Tracheal Tubes Used for Paediatric Tracheal Intubation. Pediatric Anesthesia, 11, 277-281. https://doi.org/10.1046/j.1460-9592.2001.00689.x

[5] Weiss, M., Dullenkopf, A., Fischer, J.E., Keller, C., Gerber, A.C. and European Paediatric Endotracheal Intubation Study Group (2008) Prospective Randomized Controlled Multi-Centre Trial of Cuffed or Uncuffed Endotracheal Tubes in Small Children. British Journal of Anaesthesia, 103, 867-873. https://doi.org/10.1093/bja/aep290

[6] Newth, C.J., Rachman, B. and Patel, H.J. (2004) The Use of Cuffed Versus Uncuffed Endotracheal Tubes in Pediatric Intensive Care. Journal of Pediatrics, 144, 333-337. https://doi.org/10.1016/j.jpeds.2003.12.018

[7] De Orange, F.A., Andrade, R.G., Lemos, A., Borges, P.S., Figueiroa, J.N. and Kovatsis, P.G. (2007) Cuffed versus Uncuffed Endotracheal Tubes for General Anaesthesia in Children Aged Eight Years and under. Cochrane Database of Systematic Reviews, 11, CD011954.

[8] Weiss, M., Dullenkopf, A., Gysin, C., Dillier, C.M. and Gerber, A.C. (2004) Shortcomings of Cuffed Paediatric Tracheal Tubes. British Journal of Anaesthesia, 92, 
78-88. https://doi.org/10.1093/bja/aeh023

[9] Luten, R.C., Wears, R.L., Broselow, J., Zaritsky, A., Barnett, T.M. and Lee, T. (1992) Length-Based Endotracheal Tube and Emergency Equipment in Pediatrics. Annals of Emergency Medicine, 21, 900-904. https://doi.org/10.1016/S0196-0644(05)82924-5

[10] Orf, J., Thomas, S.H., Ahmed, W., Wiebe, L., Chamberlin, P., Wedel, S.K., et al. (2000) Appropriateness of Endotracheal Tube Size and Insertion Depth in Children Undergoing Air Medical Transport. Pediatric Emergency Care, 16, 321-327. https://doi.org/10.1097/00006565-200010000-00004

[11] American Heart Association, American Academy of Pediatrics (2005) American Heart Association (AHA) Guidelines for Cardiopulmonary Resuscitation (CPR) and Emergency Cardiovascular Care (ECC) of Pediatric and Neonatal Patients: Neonatal Resuscitation Guidelines. Pediatrics, 117, e989-1004.

[12] Phipps, L.M., Thomas, N.J., Gilmore, R.K., Raymond, J.A., Bittner, T.R., Orr, R.A., et al. (2005) Prospective Assessment of Guidelines for Determining Appropriate Depth of Endotracheal Tube Placement in Children. Pediatric Critical Care Medicine, 6, 519-522. https://doi.org/10.1097/01.PCC.0000165802.32383.9E

[13] Lau, N., Playfor, S.D., Rashid, A. and Dhanarass, M. (2006) New Formulae for Predicting Tracheal Tube Length. Pediatric Anesthesia, 16, 1238-1243. https://doi.org/10.1111/j.1460-9592.2006.01982.x

[14] Luten, R. (2005) Accurate Endotracheal Tube Placement in Children: Depth of Insertion Is Part of a Process. Pediatric Critical Care Medicine, 6, 606-608. https://doi.org/10.1097/01.PCC.0000175992.99776.9D

[15] Kemper, M., Imach, S., Buehler, P.K., Thomas, J., Dave, M. and Weiss, M. (2018) Tube Tip and Cuff Positon Using Different Strategies for Placement of Currently Available Paediatric Tracheal Tubes. British Journal of Anaesthesia, 490-495. https://doi.org/10.1016/j.bja.2018.05.002

[16] Verghese, S.T., Hannallah, R.S., Patel, K.M., Cross, R.R. and Patel, K.M. (2004) Auscultation of Bilateral Breath Sounds Does Not Rule out Endobronchial Intubation in Children. Anesthesia \& Analgesia, 99, 56-58. https://doi.org/10.1213/01.ANE.0000118104.23660.F3

[17] Weiss, M., Knirsch, W., Kretschmar, O., Dullenkopf, A., Tomaske, M., Balmer, C., et al. (2006) Tracheal Tube-Tip Displacement in Children during Head Neck Movement a Radiological Assessment. British Journal of Anaesthesia, 96, 486-491. https://doi.org/10.1093/bja/ael014

[18] Böttcher-Haberzeth, S., Dullenkopf, A., Gitzelmann, C.A. and Weiss, M. (2007) Tracheal Tube Tip Displacement during Laparoscopy in Children. Anaesthesia, 62, 131-134. https://doi.org/10.1111/j.1365-2044.2006.04892.x 